\title{
Hemophagocytic Lymphohistiocytosis Secondary to PD-1 and IDO Inhibition in a Patient with Refractory Glioblastoma
}

\author{
Rohit Thummalapalli ${ }^{a}$ Thatcher Heumann ${ }^{a}$ Julie Stein ${ }^{b}$ Sarah Khan ${ }^{e}$ \\ David S. Priemer $^{\mathrm{b}}$ Amy S. Duffield ${ }^{\mathrm{b}}$ John Laterra ${ }^{\mathrm{c}}$ Rima Couzi $^{\mathrm{a}}$ \\ Michael Lim ${ }^{d}$ Matthias Holdhoff ${ }^{a}$ \\ aDepartment of Oncology, The Johns Hopkins University School of Medicine and \\ The Sidney Kimmel Comprehensive Cancer Center at Johns Hopkins, Baltimore, MD, USA; \\ bDepartment of Pathology, The Johns Hopkins University School of Medicine, \\ Baltimore, MD, USA; 'Department of Neurology, The Johns Hopkins University School of \\ Medicine, Baltimore, MD, USA; ${ }^{d}$ Department of Neurosurgery, The Johns Hopkins University

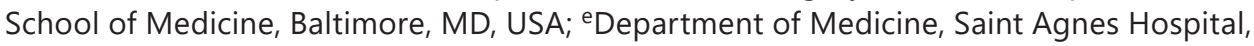 \\ Baltimore, MD, USA
}

\section{Keywords}

Hemophagocytic lymphohistiocytosis · Programmed cell death receptor-1 .

Indoleamine-pyrrole 2,3-dioxygenase - Immune checkpoint inhibition · Immune-related adverse events · Glioblastoma

\begin{abstract}
Immune checkpoint inhibition (ICI)-based approaches have transformed the treatment landscape of numerous solid tumors. Glioblastoma (GBM) is an aggressive and almost universally fatal disease which is in need of novel treatment options, and combinations of immune checkpoint inhibitors, including dual agent therapy, are starting to be explored in refractory GBM. Growing adoption of $\mathrm{ICl}$-based approaches in solid tumors has been met with improved understanding of immune-related adverse events (IRAEs), including primary hematologic adverse events. Although management guidelines for multiple hematologic IRAEs have been established, the emergence of hemophagocytic lymphohistiocytosis $(\mathrm{HLH})$ secondary to $\mathrm{ICI}$ therapy has only rarely been described, and its pathogenesis and optimal management are incompletely understood. We present the case of a 74-year-old male with a history of refractory GBM treated with PD-1 and indoleamine-pyrrole 2,3-dioxygenase (IDO) inhibition who experienced acute liver injury, followed by progressive fevers, altered mental status, and cytopenias. Serum studies and examination of spleen and bone marrow pathology were consistent with $\mathrm{HLH}$, which was refractory to steroids and ultimately resulted in his rapid clinical decline. Here, we review prior cases of HLH secondary to ICI therapy across solid tumors, and explore potential mechanisms contributing to the rapid onset and refractory nature of our
\end{abstract}


Thummalapalli et al.: Hemophagocytic Lymphohistiocytosis after Immune Checkpoint Inhibition

patient's HLH syndrome. We hope to further highlight HLH as an emerging hematologic IRAE secondary to $\mathrm{ICl}$ therapy, and suggest that new practice guidelines begin to recognize $\mathrm{HLH}$ as a characteristic hematologic IRAE in patients treated with PD-1 and other immune checkpoint inhibitors.

(C) 2020 The Author(s)

Published by S. Karger AG, Basel

\section{Background}

Immune checkpoint inhibition (ICI) can produce durable responses in subsets of solid tumor patients, and is therefore starting to be widely explored across cancers. Glioblastomas are the most common primary brain cancers in adults, and despite aggressive multimodal management, virtually all patients eventually face recurrence and die of their disease. In this setting, there has been a strong interest in exploring immunotherapeutic treatments for patients with glioblastomas. ICI therapy is classically associated with characteristic immunerelated adverse events (IRAEs), including colitis, hepatitis, pneumonitis, and endocrinopathies [1]. However, the emergence of new and less common IRAEs, including hematologic toxicities, continues to be explored, particularly in the setting of dual ICI therapy and clinical trials of novel ICI agents. Management of hematologic IRAEs including autoimmune hemolytic anemia, acquired TTP/hemolytic uremic syndrome, aplastic anemia, immune thrombocytopenia, and acquired hemophilias have been described in recent practice guidelines [2]; however, the presentation and management of hemophagocytic lymphohistiocytosis (HLH) secondary to ICI therapy has yet to be rigorously explored or described in practice guidelines. Recently, several reports have described HLH in solid tumor patients treated with ICI [3-11]; these HLH syndromes varied in terms of method of diagnosis, onset, severity, and response to immunosuppressive modalities. In this case report, we describe a patient with recurrent glioblastoma who developed HLH while on a clinical trial with the PD-1 inhibitor nivolumab and a novel indoleamine-pyrrole 2,3-dioxygenase (IDO) inhibitor.

\section{Case Presentation}

A 74-year-old male with a history of glioblastoma, coronary artery disease, atrial fibrillation, hypertension, hyperlipidemia, and type 2 diabetes mellitus presented to our service with abnormal liver enzymes found at an outpatient clinic visit. Thirteen months prior to admission, he had developed aphasia resulting from a left temporal lobe enhancing mass found on imaging. Subsequent surgical resection revealed a BRAF V600E mutated, IDH1 wild type, MGMT promoter unmethylated glioblastoma. His disease progressed following 6 weeks of fractionated radiation with concurrent temozolomide and four cycles of monthly adjuvant temozolomide. He was then enrolled in a phase I trial of nivolumab and anti-IDO immunotherapy for patients with first glioblastoma recurrence (NCT03707457). He received a single infusion of nivolumab, and then was started on monthly nivolumab and once daily BMS-986205, an oral ID01 inhibitor. On cycle 2, day 17 of nivolumab and BMS-986205, he was found to have an elevated AST of 832, ALT of 1,378, alkaline phosphatase of 152, and total bilirubin of 4.1, and was admitted to the inpatient service. Duplex liver ultrasound, CT imaging, and markers for autoimmune, infiltrative, and viral etiologies of liver injury proved unremarkable. As a result of this negative workup, he was treated for immune-mediated hepatitis, secondary to his anti-PD-1 and/or anti-IDO therapy, and was initiated on IV methylprednisolone. His liver enzymes continued to uptrend to a peak of AST 1,064, ALT 1,675 on day four of admission leading to an increase in steroid dosing followed by a liver biopsy. Pathology was significant 
Thummalapalli et al.: Hemophagocytic Lymphohistiocytosis after Immune Checkpoint Inhibition

Fig. 1. Histologic examination of the spleen, showing infiltration of foamy histocytes containing occasional hematolymphoid cells.

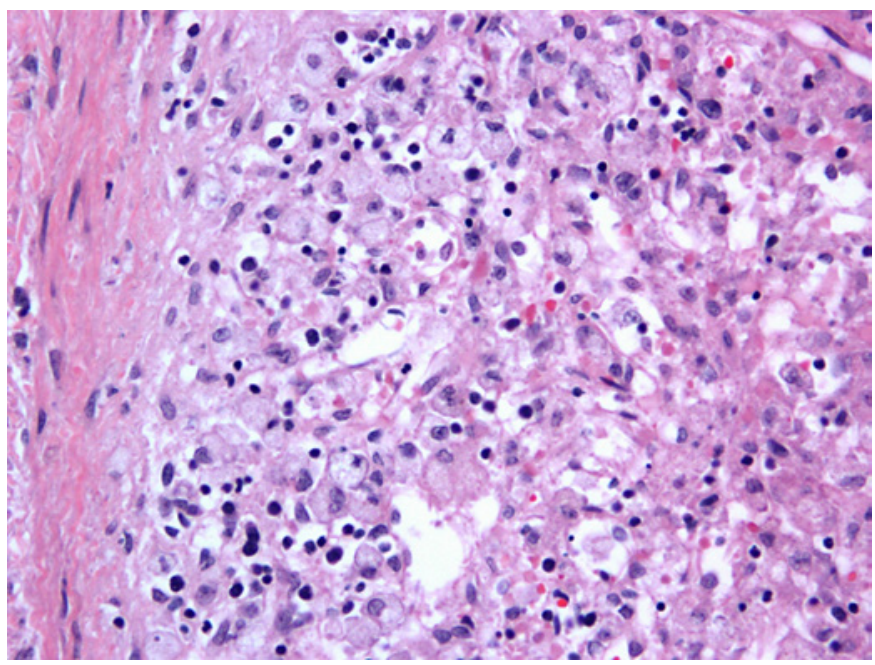

for focal bile duct injury, mild portal inflammation, and minimal lymphocytic lobular infiltration. Overall, these findings were non-specific but possibly supportive of a resolving hepatitis. His transaminases began to downtrend and he was weaned to oral prednisone.

On day 8 of admission, he started to experience new fevers, progressively worsening mental status, new leukopenia to a total WBC of 460 per $\mathrm{mm}^{3}$, and new neutropenia to an absolute neutrophil count of 350 per $\mathrm{mm}^{3}$. His worsening mental status was not thought to be reflective of worsening liver dysfunction or steroid-related delirium, and was concerning for an infectious or inflammatory cause. He underwent a lumbar puncture which showed 18 WBCs per $\mathrm{mm}^{3}$ (95\% lymphocytes), a normal glucose of $61 \mathrm{mg} / \mathrm{dL}$, and a slightly elevated total protein of $74.4 \mathrm{mg} / \mathrm{dL}$, which raised some concern for an autoimmune encephalitis that had been unresponsive to steroids. CSF was positive for malignant cells. A broad serum, urine, and CSF infectious workup was sent but was only significant for low level serum and urine BK virus, low level serum JC viremia, and negative JC virus in CSF; his clinical syndrome was not thought to be consistent with BKV meningoencephalitis. CT chest, abdomen, and pelvis showed mild duodenal and perinephric fat stranding, an irregular posterior bladder wall with a hyperattenuating nodule, and splenomegaly to $15.6 \mathrm{~cm}$. Given the progressive neutropenia and fevers, he was broadly covered with IV vancomycin, ceftazidime, metronidazole, amphotericin B, acyclovir, and atovaquone.

Given the progressive cytopenias, fevers, altered mental status, splenomegaly, and continued bilirubin elevation without a clear alternate cause, the diagnosis of HLH was considered. Ferritin was elevated at 33,738 ng/mL (normal <400), IL-6 ELISA was elevated at $37.3 \mathrm{pg} / \mathrm{mL}$ (normal <5), and triglycerides were elevated at $843 \mathrm{mg} / \mathrm{dL}$ (normal <150). Whether this represented a true HLH process or simply reflected profound inflammation in the setting of severe hepatitis, undiagnosed infection, or other IRAE was unclear. Accounting for the patient's rapid clinical decline and lack of further safe treatment options, his family declined a bone marrow biopsy. He was ultimately transitioned to comfort measures, became progressively somnolent, and expired on day 15 of admission.

Postmortem soluble IL-2 receptor was found to be significantly elevated at 28,985 pg/ $\mathrm{mL}$ (normal 532-1,891). Postmortem autopsy was completed. Gross pathology was significant for generalized jaundice, hepatomegaly $(2,350 \mathrm{~g}$; reference range: 1,500-1,800 g), and splenomegaly (350 g; reference range: 150-200 g). Histologic examination of the spleen (Fig. 1) and bone marrow (Fig. 2) was supportive of HLH, showing scattered infiltration by foamy histiocytes which occasionally contained hematolymphoid cells and/or cell debris, 
Fig. 2. Histologic examination of the bone marrow, showing infiltration of foamy histocytes containing occasional hematolymphoid cells.

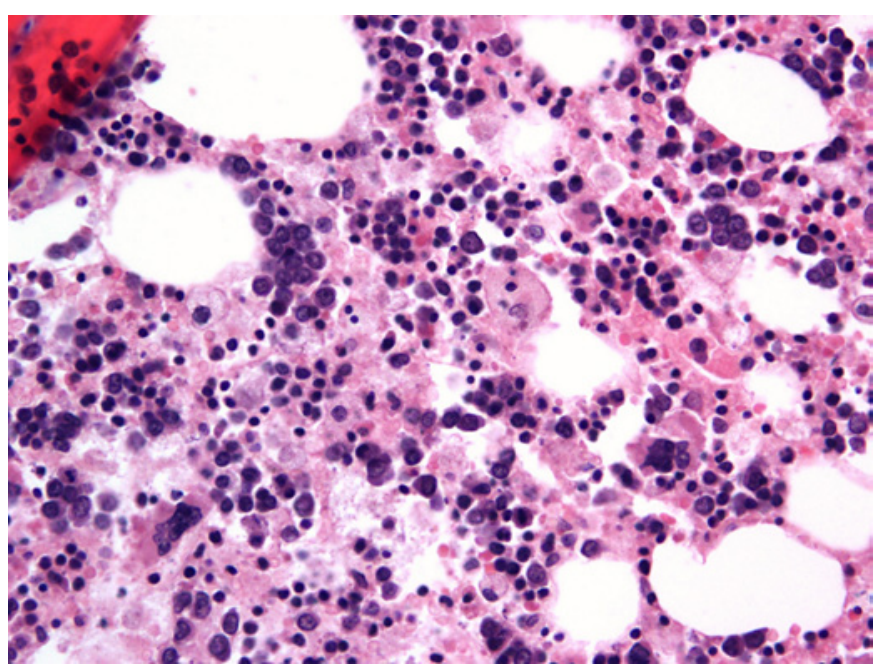

Fig. 3. Histologic examination of the liver, showing canalicular and intrahepatic cholestasis, centrilobular hepatocellular dropout, scarring of central veins, and mild mixed portal inflammation and foci of lobular inflammation.

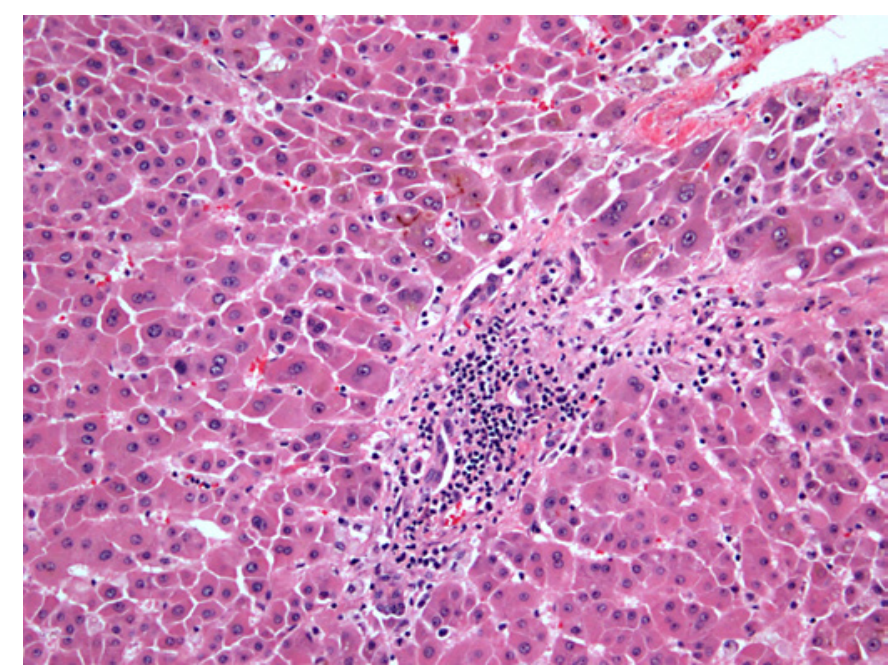

consistent with hemophagocytosis. His final HScore was 239, reflecting a 98-99\% probability of HLH. Histologic examination of the liver showed canalicular and intrahepatic cholestasis (Fig. 3). There was also centrilobular hepatocellular dropout, which was seen in coordination with scarring and/or distortion of the central veins. It was difficult to characterize the etiology of the central vein injury, but it may have represented the effects of a prior vasculitis. There was only mild mixed portal inflammation and rare foci of lobular inflammation, which was unconvincing for an antecedent ICI-induced autoimmune hepatitis, and there was no evidence of HLH in the liver (Fig. 3). Hence, the liver pathology was not consistent with either HLH or ICI-induced autoimmune hepatitis, and perhaps could have been secondary to sinusoidal congestion from drug-induced liver injury, or an incompletely characterized vasculitis.

\section{Discussion/Conclusions}

HLH represents a rare, severe inflammatory reaction characterized by overstimulation of lymphocytes and macrophages, and is characterized by high levels of serum cytokines, the presence of hemophagocytosis in the reticuloendothelial and lymphoid systems, and often 
Thummalapalli et al.: Hemophagocytic Lymphohistiocytosis after Immune Checkpoint Inhibition

Table 1. List of prior cases of HLH secondary to ICI therapy

\begin{tabular}{|c|c|c|c|c|c|c|c|}
\hline Reference & Type of ICI & $\begin{array}{l}\text { Timing/cycles of } \\
\text { therapy }\end{array}$ & $\begin{array}{l}\text { Primary } \\
\text { malignancy }\end{array}$ & Method of diagnosis & $\begin{array}{l}\text { BM biopsy/ } \\
\text { pathology }\end{array}$ & Treatment & $\begin{array}{l}\text { Clinical } \\
\text { outcome }\end{array}$ \\
\hline $\begin{array}{l}\text { Takeshita et al., } \\
2017 \text { [3] }\end{array}$ & Nivolumab & 2 doses & NSCLC & BM biopsy & BM biopsy + for HLH & Steroids & Improvement \\
\hline $\begin{array}{l}\text { Malissen et al., } \\
2017 \text { (case 1) [4] }\end{array}$ & Nivolumab & 17 months & Melanoma & BM biopsy & BM biopsy + for HLH & Steroids & Death \\
\hline $\begin{array}{l}\text { Honjo et al., } \\
2019[10]\end{array}$ & Nivolumab & 4 doses & NSCLC & $\begin{array}{l}\text { Ferritin, soluble IL-2R, } \\
\text { triglyceride elevation }\end{array}$ & $\mathrm{N} / \mathrm{A}$ & $\begin{array}{l}\text { Steroids, } \\
\text { mycophenolate } \\
\text { mofetil }\end{array}$ & Improvement \\
\hline $\begin{array}{l}\text { Sadaat and Jang, } \\
2018 \text { [9] }\end{array}$ & Pembrolizumab & 6 doses & Melanoma & $\begin{array}{l}\text { NK cell functional assay, } \\
\text { soluble CD163 elevation }\end{array}$ & $\mathrm{N} / \mathrm{A}$ & Steroids & Improvement \\
\hline $\begin{array}{l}\text { Shah et al., } \\
2017 \text { [5] }\end{array}$ & Pembrolizumab & 9 months & $\begin{array}{l}\text { Bladder } \\
\text { cancer }\end{array}$ & $\begin{array}{l}\text { BM biopsy, NK cell } \\
\text { functional assay, soluble } \\
\text { IL-2R elevation }\end{array}$ & BM biopsy + for HLH & $\begin{array}{l}\text { Steroids and } \\
\text { etoposide } \\
\text { (HLH 2004) }\end{array}$ & $?$ \\
\hline $\begin{array}{l}\text { Okawa et al., } \\
2019[6]\end{array}$ & Pembrolizumab & 1 dose & NSCLC & $\begin{array}{l}\text { BM biopsy, soluble IL-2R, } \\
\text { ferritin elevation }\end{array}$ & BM biopsy + for HLH & Steroids & Improvement \\
\hline $\begin{array}{l}\text { Laderian et al., } \\
2019 \text { [7] }\end{array}$ & Pembrolizumab & 12 months & $\begin{array}{l}\text { Thymic } \\
\text { cancer }\end{array}$ & $\begin{array}{l}\text { BM biopsy, liver biopsy, } \\
\text { soluble IL-2R, ferritin } \\
\text { elevation, cytopenias }\end{array}$ & BM biopsy + for HLH & $\begin{array}{l}\text { Steroids, } \\
\text { IVIG, anakinra }\end{array}$ & Death \\
\hline $\begin{array}{l}\text { Malissen et al., } \\
2017 \text { (case 2) [4] }\end{array}$ & Ipilimumab & $\begin{array}{l}1 \text { dose of ipilimumab; } \\
\text { prior history of } \\
9 \text { months of nivolumab }\end{array}$ & Melanoma & $\begin{array}{l}\text { Ferritin, triglyceride } \\
\text { elevation, cytopenias }\end{array}$ & $\begin{array}{l}\text { BM biopsy negative } \\
\text { for HLH }\end{array}$ & Steroids & Improvement \\
\hline $\begin{array}{l}\text { Malissen et al., } \\
2017 \text { (case 3) [4] }\end{array}$ & Avelumab & 1 dose & $\begin{array}{l}\text { Merkel cell } \\
\text { carcinoma }\end{array}$ & $\begin{array}{l}\text { Ferritin, triglyceride } \\
\text { elevation, cytopenias }\end{array}$ & $\mathrm{N} / \mathrm{A}$ & Steroids & Death \\
\hline $\begin{array}{l}\text { Hantel et al., } \\
2018 \text { [8] }\end{array}$ & $\begin{array}{l}\text { Ipilimumab and } \\
\text { nivolumab }\end{array}$ & $\begin{array}{l}4 \text { doses of ipilimumab, } \\
1 \text { dose of ipilimumab } \\
\text { and nivolumab } \\
\text { ( } 3 \text { weeks prior) }\end{array}$ & Melanoma & $\begin{array}{l}\text { BM biopsy, soluble IL-2R } \\
\text { elevation }\end{array}$ & BM biopsy + for HLH & Steroids & Improvement \\
\hline $\begin{array}{l}\text { Satzger et al., } \\
2018[11]\end{array}$ & $\begin{array}{l}\text { Ipilimumab and } \\
\text { nivolumab }\end{array}$ & 4 doses & Melanoma & $\begin{array}{l}\text { Liver biopsy, ferritin, } \\
\text { triglyceride, soluble IL-2 } \\
\text { elevation, cytopenias }\end{array}$ & $\mathrm{N} / \mathrm{A}$ & $\begin{array}{l}\text { Steroids, } \\
\text { mycophenolate } \\
\text { mofetil }\end{array}$ & Improvement \\
\hline
\end{tabular}

results in multi-organ failure, particularly of the liver and bone marrow [12]. HLH can be primary (genetic cause, usually presenting in childhood) or secondary to a number of causes including infections (particularly viral including EBV, as well as bacterial, and fungal), hematologic malignancies (particularly of T and NK lineages), autoimmune conditions (including SLE and adult-onset Still's disease), immunodeficient states (including chemotherapy or modes of immune suppression) [12], and is starting to be described in patients receiving ICI therapy.

We found a total of six pathology-proven cases of HLH in the setting of ICI for solid tumors, including nivolumab [3,4], pembrolizumab [5-7], and combined ipilimumab and nivolumab [8] (Table 1). We also found an additional five cases [4, 9-11] which were diagnosed based on serum findings, including elevated ferritin, soluble IL-2 receptor, triglycerides, NK cell functional assays, and cytopenias (Table 1). The majority of patients were receiving ICI for melanoma or NSCLC (eight of 11 cases); no prior cases were described in patients with glioblastoma. All 11 patients received steroids. Three received additional immunosuppression, including two with mycophenolate mofetil $[10,11]$, and one with IVIG and anakinra [7]. One received etoposide according to HLH-2004 protocol [5]. In contrast to our patient, five of seven showed clinical improvement with steroids alone $[3,4,6,8,9]$; in addition, our patient experienced HLH earlier than all cases except two, with one case occurring after one dose of pembrolizumab [6] and another after one dose of avelumab [4]. Overall, this suggested that our patient may have had a particularly aggressive manifestation 
of the syndrome, and we wondered whether concurrent ID01 inhibition could have contributed. Upregulation of the IDO pathway has been implicated as an escape mechanism to PD-1 blockade, and acts by metabolizing tryptophan to kynurenine, which can promote differentiation of $\mathrm{T}$ regulatory cells and induction of $\mathrm{T}$ cell tolerance [13]. However, patients with HLH have been shown to have high plasma kynurenine to tryptophan ratios [14], and ID01 knockout murine models have not been shown to affect clinical outcome in secondary HLH models [15]. This suggested that PD-1 blockade was likely the main driver of our patient's immunotoxicity.

\section{Conclusions}

Taken together, this case represents a particularly early and aggressive form of HLH secondary to combination ICI therapy, and the first described in a patient receiving combined PD-1 and IDO inhibition. As new ICI agents continue to be explored in the management of solid tumors, the increasing incidence of HLH as a possible severe IRAE should be considered, and practice guidelines should begin to recognize HLH is a more commonly observed hematologic IRAE.

\section{Acknowledgement}

We thank the patient's family for agreeing to the report of his case.

\section{Statement of Ethics}

The study (ClinicalTrials.gov ID: NCT03707457) was approved by the Institutional Review Board (IRB) of the Johns Hopkins Medical Institutions. Informed consent for participation in the clinical trial (ClinicalTrials.gov ID: NCT03707457) had been obtained by the patient. Consent for publication was obtained from the family.

\section{Disclosure Statement}

M.H. has consulted or served on an advisory board for Celgene, Abbvie, BTG International, and NewLink Genetics. M.L. has research support from Arbor, BMS, Accuray, DNAtrix, Tocagen, Biohaven, and Kyrin-Kyowa, and has consulted for Tocagen, SQZ Technologies, VBI, and Stryker. The authors declare that there are no competing interests.

\section{Funding Sources}

This study was supported by the Sidney Kimmel Comprehensive Cancer Center Core Grant No. P30CA006973. 
Thummalapalli et al.: Hemophagocytic Lymphohistiocytosis after Immune Checkpoint Inhibition

\section{Author Contributions}

R.T., T.H., S.K., R.C., J.L., R.C., M.L., and M.H. took clinical care of the patient. J.S., D.S.P., and A.S.D. provided pathology figures and interpreted pathology specimens. R.T. wrote the manuscript with input from all authors. All authors read and approved the final manuscript. M.H. reviewed and interpreted the data presented in this case report, coordinated this project, contributed to writing the manuscript, and has approved the final manuscript.

\section{References}

1 Topalian SL, Hodi FS, Brahmer JR, Gettinger SN, Smith DC, McDermott DF, et al. Safety, activity, and immune correlates of anti-PD-1 antibody in cancer. N Engl J Med. 2012;366(26):2443-54.

2 Brahmer JR, Lacchetti C, Thompson JA, Atkins MB, Brassil KJ, Caterino JM, et al.; National Comprehensive Cancer Network. Management of immune-related adverse events in patients treated with immune checkpoint inhibitor therapy: American society of clinical oncology clinical practice guideline summary. J Oncol Pract. 2018;14(4):247-9.

3 Takeshita M, Anai S, Mishima S, Inoue K. Coincidence of immunotherapy-associated hemophagocytic syndrome and rapid tumor regression. Ann Oncol. 2017;28(1):186-9.

4 Malissen N, Lacotte J, Du-Thanh A, Gaudy-Marqueste C, Guillot B, Grob JJ. Macrophage activation syndrome: a new complication of checkpoint inhibitors. Eur J Cancer. 2017;77:88-9.

5 Shah D, Shrestha R, Ramlal R, Hatton J, Saeed H. Pembrolizumab associated hemophagocytic lymphohistiocytosis. Ann Oncol. 2017;28(6):1403.

6 Okawa S, Kayatani H, Fujiwara K, Ozeki T, Takada K, Iwamoto Y, et al. Pembrolizumab-induced autoimmune hemolytic anemia and hemophagocytic lymphohistiocytosis in non-small cell lung cancer. Intern Med. 2019; 58(5):699-702.

7 Laderian B, Koehn K, Holman C, Lyckholm L, Furqan M. Association of hemophagocytic lymphohistiocytosis and programmed death 1 checkpoint inhibitors. J Thorac Oncol. 2019;14(4):e77-8.

8 Hantel A, Gabster B, Cheng JX, Golomb H, Gajewski TF. Severe hemophagocytic lymphohistiocytosis in a melanoma patient treated with ipilimumab + nivolumab. J Immunother Cancer. 2018;6(1):73.

9 Sadaat M, Jang S. Hemophagocytic lymphohistiocytosis with immunotherapy: brief review and case report. J Immunother Cancer. 2018;6(1)49.

10 Honjo O, Kubo T, Sugaya F, Nishizaka T, Kato K, Hirohashi Y, et al. Severe cytokine release syndrome resulting in purpura fulminans despite successful response to nivolumab therapy in a patient with pleomorphic carcinoma of the lung: a case report. J Immunother Cancer. 2019;7(1):97.

11 Satzger I, Ivanyi P, Länger F, Kreipe HH, Schaper-Gerhardt K, Beutel G, et al. Treatment-related hemophagocytic lymphohistiocytosis secondary to checkpoint inhibition with nivolumab plus ipilimumab. Eur J Cancer. 2018;93:150-3.

12 Jordan MB, Allen CE, Weitzman S, Filipovich AH, McClain KL. How I treat hemophagocytic lymphohistiocytosis. Blood. 2011;118(15):4041-52.

13 Moon YW, Hajjar J, Hwu P, Naing A. Targeting the indoleamine 2,3-dioxygenase pathway in cancer. J Immunother Cancer. 2015;3:51:

14 Put K, Avau A, Brisse E, Mitera T, Put S, Proost P, et al. Cytokines in systemic juvenile idiopathic arthritis and haemophagocytic lymphohistiocytosis: tipping the balance between interleukin-18 and interferon- $\gamma$. Rheumatology (Oxford). 2015;54(8):1507-17.

15 Put K, Brisse E, Avau A, Imbrechts M, Mitera T, Janssens R, et al. Ido1 deficiency does not affect disease in mouse models of systemic juvenile idiopathic arthritis and secondary hemophagocytic lymphohistiocytosis. PLoS ONE. 2016;11(2):e0150075, 